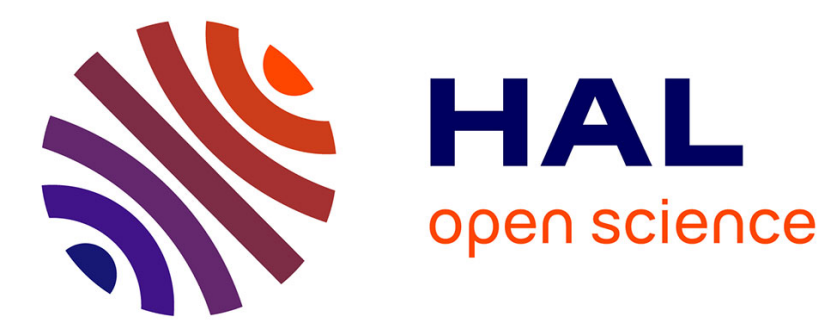

\title{
Ferroelectric control of magnetic domains in ultra-thin cobalt layers
}

\author{
Z Huang, S Stolichnov, Anne Bernand-Mantel, J. Borrel, S Auffret, Gilles \\ Gaudin, O Boulle, S. Pizzini, L. Ranno, L Herrera Diez, et al.
}

\section{- To cite this version:}

Z Huang, S Stolichnov, Anne Bernand-Mantel, J. Borrel, S Auffret, et al.. Ferroelectric control of magnetic domains in ultra-thin cobalt layers. Applied Physics Letters, 2013, 103 (22), pp.222902. 10.1063/1.4833495 . hal-01444801

\section{HAL Id: hal-01444801 \\ https://hal.science/hal-01444801}

Submitted on 24 Jan 2017

HAL is a multi-disciplinary open access archive for the deposit and dissemination of scientific research documents, whether they are published or not. The documents may come from teaching and research institutions in France or abroad, or from public or private research centers.
L'archive ouverte pluridisciplinaire HAL, est destinée au dépôt et à la diffusion de documents scientifiques de niveau recherche, publiés ou non, émanant des établissements d'enseignement et de recherche français ou étrangers, des laboratoires publics ou privés. 


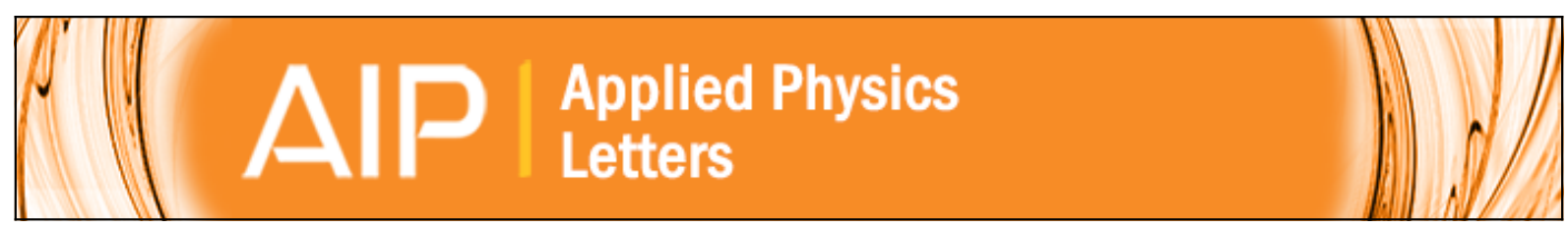

\section{Ferroelectric control of magnetic domains in ultra-thin cobalt layers}

Z. Huang, I. Stolichnov, A. Bernand-Mantel, J. Borrel, S. Auffret, G. Gaudin, O. Boulle, S. Pizzini, L. Ranno, L. Herrera Diez, and N. Setter

Citation: Applied Physics Letters 103, 222902 (2013); doi: 10.1063/1.4833495

View online: http://dx.doi.org/10.1063/1.4833495

View Table of Contents: http://scitation.aip.org/content/aip/journal/apl/103/22?ver=pdfcov

Published by the AIP Publishing

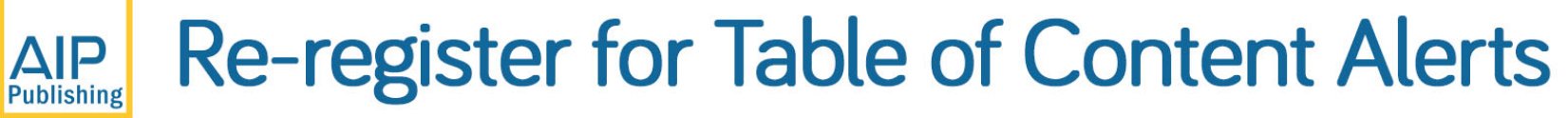




\title{
Ferroelectric control of magnetic domains in ultra-thin cobalt layers
}

\author{
Z. Huang, ${ }^{1}$ I. Stolichnov, ${ }^{1}$ A. Bernand-Mantel, ${ }^{2,3}$ J. Borrel,,${ }^{2,3}$ S. Auffret, ${ }^{4}$ G. Gaudin, ${ }^{4}$ \\ O. Boulle, ${ }^{4}$ S. Pizzini, ${ }^{2,3}$ L. Ranno, ${ }^{2,3}$ L. Herrera Diez, ${ }^{2,3}$ and N. Setter ${ }^{1}$ \\ ${ }^{1}$ Ceramics Laboratory, EPFL-Swiss Federal Institute of Technology, Lausanne 1015, Switzerland \\ ${ }^{2}$ University of Grenoble Alpes, Institut Néel, F-38042 Grenoble, France \\ ${ }^{3}$ CNRS, Institut Néel, F-38042 Grenoble, France \\ ${ }^{4}$ SPINTEC, UMR-8191, CEA/CNRS/UJF/GINP, INAC, F-38054 Grenoble, France
}

(Received 11 September 2013; accepted 28 October 2013; published online 25 November 2013)

\begin{abstract}
Non-volatile ferroelectric control of magnetic domains has been demonstrated in ultra-thin cobalt layers at room temperature. The sensitivity of magnetic anisotropy energy to the electronic structure in a few atomic layers adjacent to the interface allows for ferroelectric control of coercivity and magnetic domain dynamics. These effects have been monitored and quantified using magnetooptical Kerr effect. In particular, the regimes, where the ferroelectric domains enhance/inhibit the magnetic domain nucleation or increase/reduce domain wall velocity, have been explored. Thus, non-destructive and reversible ferroelectric domain writing provides a tool to define the magnetic domain paths, create nucleation sites, or control domain movement. (C) 2013 AIP Publishing LLC. [http://dx.doi.org/10.1063/1.4833495]
\end{abstract}

The systems with magnetoelectric coupling, where the magnetic properties are controlled via the external electric field, have been intensively explored within the last decade, because of their potential for information storage and processing. There is a focus on multilayer structures incorporating ultra-thin magnetic channels and dielectric layers, which is the most suitable architecture for the field effect control of ferromagnetism. Such structures have been achieved first using diluted magnetic semiconductors like $(\mathrm{Ga}, \mathrm{Mn}) \mathrm{As}$ and (In,Mn)As, where the ferromagnetism is mediated by strongly spin-orbit coupled holes. ${ }^{1,2}$ Replacing the conventional dielectric in such heterostructures with ferroelectric material results in a multiferroic system that offers additional functionalities of non-volatile switching and non-destructive writing of ferroelectric domains. ${ }^{3,4}$ However, the low Curie temperature $\left(\mathrm{T}_{\mathrm{C}}\right)$ of ferromagnetic phase transition in diluted magnetic semiconductors remains a major obstacle for practical use of these results.

Recently, significant electric-field-driven modulation of $\mathrm{T}_{\mathrm{C}}$ and coercivity has been demonstrated at room temperature in heterostructures with ultra-thin layers of conventional ferromagnetic metals. ${ }^{5-9}$ In such layers, a delicate balance between different contributions to the magnetic anisotropy energy (MAE) results in their high sensitivity to the changes of electronic structure at the interface. ${ }^{10}$ Thus, a weak change of charge density induced by the electric field at the extremely narrow interface-adjacent layer is sufficient for a substantial change of coercivity, ${ }^{5-7} \mathrm{~T}_{\mathrm{C}},{ }^{8,9}$ and magnetic domain kinetics. ${ }^{11,12}$ In context of these findings, the ferroelectric/ferromagnetic heterostructures become of interest for electronic elements operational within the conventional temperature range. First principles calculations performed for multilayers combining ultra-thin Co layers and P(VDF-TrFE) (polyvinylidene fluoride-trifluoroethylene) ferroelectric copolymer confirm an appreciable multiferroic coupling. ${ }^{13}$ These results agree with the report of significant magnetic anisotropy change that has been experimentally detected in this system using magneto-optical Kerr effect (MOKE) analysis. ${ }^{14}$
One of the most interesting features offered by the structures combining ferroelectricity and ferromagnetism is a possibility to influence the magnetic properties locally by electrical writing of stable ferroelectric domains. ${ }^{15,16}$ Here, we address this topic focusing on the structures with fieldeffect-mediated local control of magnetic domain nucleation and growth in ultra-thin Co layer at room temperature.

The multiferroic heterostructure used for this study is schematically represented in Fig. 1(a). The multilayer consisting of $\mathrm{Pt}(3 \mathrm{~nm}) / \mathrm{Co}(0.6 \mathrm{~nm}) / \mathrm{Al}$ (wedge $\sim 1.5 \mathrm{~nm}$ ) stack was deposited by sputtering on Si substrate. ${ }^{10}$ Following the deposition, the top $\mathrm{Al}$ layer was treated by oxygen plasma in order to reach a partially oxidized $\mathrm{AlO}_{\mathrm{x}}$ layer. The gradually changing Al thickness along the wedge with the rate of $1 \AA / \mathrm{cm}$ leads to the continuous variation of the $\mathrm{Co} / \mathrm{AlO}_{\mathrm{x}}$ interface oxidation degree. The magnetic properties of the Co layer are known to be very sensitive to the $\mathrm{Co} / \mathrm{AlOx}$ interface oxidation state in this system. In particular, the MAE varies along the wedge as the oxidation degree changes. ${ }^{10,17}$ For optimum oxidation, the MAE shows a maximum and the easy magnetization axis is out of plane. For this experiment, we have selected a region of the wedge near this optimum oxidation state.

The ferroelectric gate was formed by deposition of $200 \mathrm{~nm}$ P(VDF-TrFE) co-polymer layer with molar ratio $77 / 23$ via spin-coating using $2.5 \%$ methyl ethyl ketone solution. Due to the very low crystallization temperature of $130-135^{\circ} \mathrm{C}$, this ferroelectric material offers perfect processing compatibility with the magnetic channel. In order to monitor the magnetic domain behavior using optical methods, $100 \mathrm{~nm}$ indium tin oxide (ITO) transparent electrodes were deposited by sputtering. A $3 \mathrm{~nm}$ buffer $\mathrm{Al}_{2} \mathrm{O}_{3}$ layer was grown on top of $\mathrm{P}(\mathrm{VDF}-\mathrm{TrFE})$ by Atomic Layer Deposition prior to ITO deposition in order to avoid degradation of ferroelectricity due to the sputtering process. The $100 \mathrm{~nm}$ thick gate electrodes prepared using shadow mask had a rectangular shape with size of $550 \times 2000 \mu \mathrm{m}$. The ferroelectric gate showed polarization hysteresis loops typical for this material 
(a)

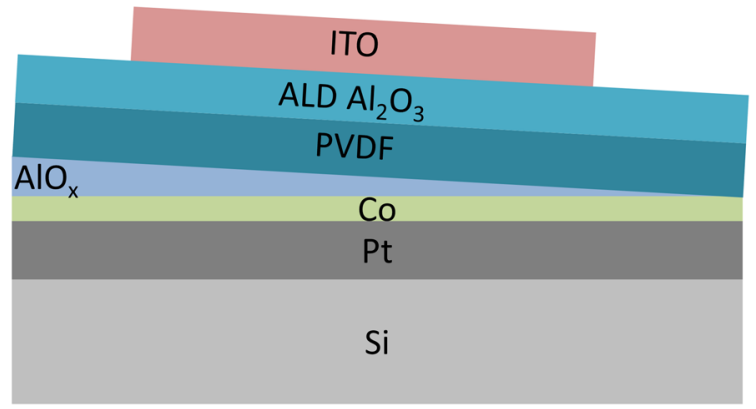

(b)

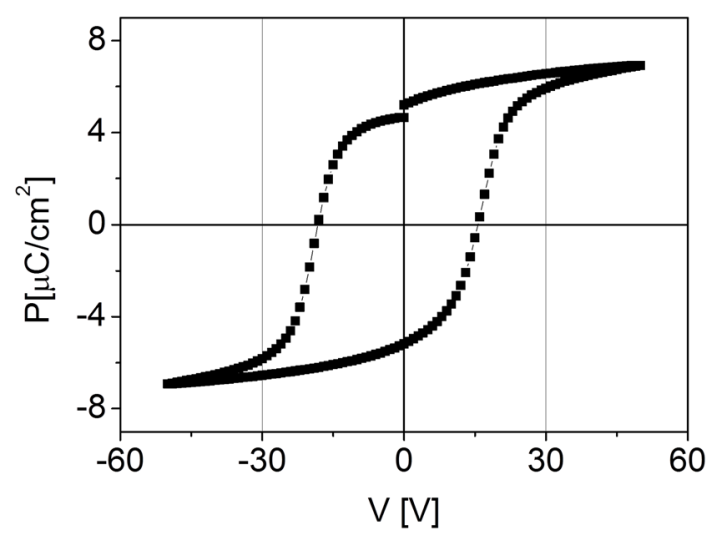

FIG. 1. (a) Cross-section of the heterostructure used for ferroelectric control of ferromagnetic domains: $\mathrm{Si} / \mathrm{Pt} / \mathrm{Co} / \mathrm{AlO}_{\mathrm{x}}$ wedge has been covered by PVDF-TrFE ferroelectric gate with $\mathrm{Al}_{2} \mathrm{O}_{3} / \mathrm{ITO}$ top electrodes. (b) Ferroelectric hysteresis loop measured on the PVDF-TrFE gate at $5 \mathrm{kHz}$ using the $\mathrm{Al}_{2} \mathrm{O}_{3} /$ ITO gate electrode.

(Fig. 1(b)), with the coercive field of $800-900 \mathrm{kV} / \mathrm{cm}$ $(16-18 \mathrm{~V})$ at $5 \mathrm{kHz}$. The ITO electrodes have been used for poling the ferroelectric layer underneath by applying voltage pulses of $\pm 30 \mathrm{~V} / 1 \mathrm{~s}$. Application of the positive voltage results in a partial (less than $1 \%$ of the electron density) accumulation of electrons in the magnetic Co layer, while the negative voltage induces the depletion effect. The time intervals between poling the ferroelectric gate and magnetic measurements varied between 5 min and 1 week. The magnetic properties of the Co layer were found to be virtually independent of the elapsed time.

The nucleation and growth of magnetic domains in the Co layer were visualized and quantified using MOKE microscopy in the polar geometry, with the magnetic field being applied out of plane. The transparency of the ITO/P(VDF-TrFE) $/ \mathrm{Al}_{2} \mathrm{O}_{3}$ gate allows us to visualize magnetic domains below the electrode. All experiments described in this letter were performed at room temperature. The coupling between the ferroelectric polarization and magnetic domain behavior is readily observed in the area with low MAE where the nucleation density is high (Figs. 2(a) and 2(b)). Poling the gate by applying to the electrode negative (positive) voltage pulse of $30 \mathrm{~V} / 1 \mathrm{~s}$ promotes (impedes) the magnetic domain nucleation. This effect is clearly seen in Figs. 2(a) and 2(b) where the MOKE image covers both gated (poled) and non-gated (non-poled) areas. The parts where magnetization is switched (non-switched) appear light grey (dark grey) in the images. Note that due to the thickness gradient, the color of central part of ITO electrodes appears darker than the peripheral areas in Fig. 2. This gradual change of brightness is also seen in the conventional optical microscopy image (inset of Fig. 2(a)) and has to be ignored because it does not correspond to any magnetic domain pattern. The vertical variation of the nucleation density is due to the anisotropy variation along the sample induced by the Al wedge. Before taking images shown in Fig. 2, the magnetic state of the sample has been fully saturated by applying a strong out of plane magnetic field. The images taken after applying the opposite magnetic field pulse of $24 \mathrm{mT} / 50 \mathrm{~ms}$ represent partially switched states and clearly show the contrast between the magnetic domains in the gated and non-gated areas. We observe a difference in both the nucleated domains' size and density. The dynamics at the origin of the magnetic domains (a)

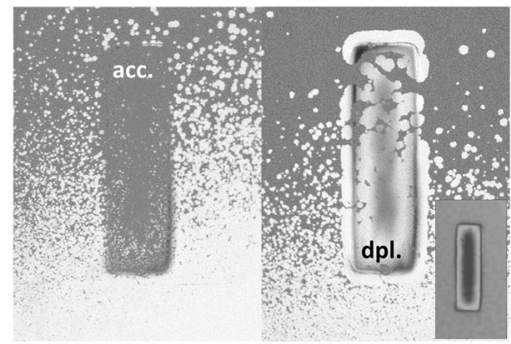

(b)
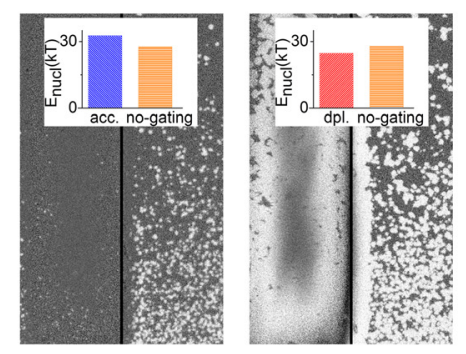

(c)

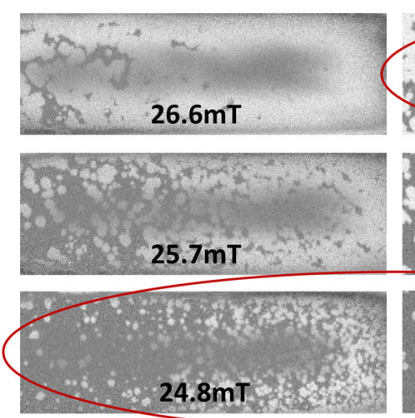

acc.

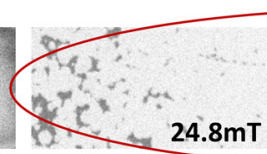

$24.8 \mathrm{mT}$
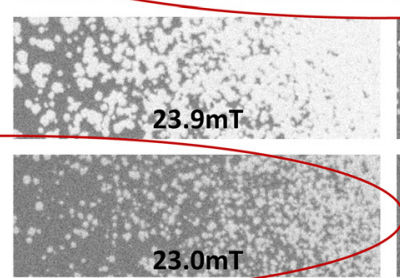

no gating
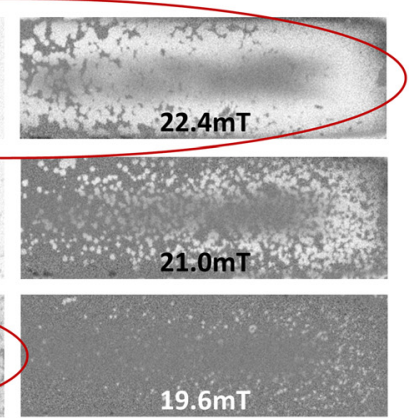

dpl.
FIG. 2. MOKE images representing the magnetization reversal in the high nucleation density regime. The density and size of new magnetic domains occurring below the rectangular gate electrode are clearly different compared to the surrounding (non-poled) areas (the image is taken after applying the magnetic field pulse of $24 \mathrm{mT} / 50 \mathrm{~ms}$ ). (a) The poled ferroelectric gate induces the effect of accumulation (left) or depletion (right). Inset: Optical image of one ITO electrode. (b) Comparison of the nucleation density below the poled ferroelectric gate and the adjacent non-poled areas. Insets: Nucleation energies extracted from the nucleation density for the corresponding areas. (c) Comparison of the MOKE images taken after $50 \mathrm{~ms}$, at different magnetic field amplitudes and identification of the pairs of images with similar nucleation densities. 
in Fig. 2 can be visualized in the Movies 1 and 2 in supplementary material, ${ }^{18}$ where images are taken versus time at constant magnetic field.

Reversing the polarization in the ferroelectric gate, we could repeatedly change the magnetic domain nucleation density under the electrode. For each studied electrode, the polarization has been reversed 4-5 times provoking the reproducible effect of increasing or reducing the magnetic domain nucleation density.

In order to quantify the ferroelectric field effect on the magnetic switching, we have carried out a comparative analysis of the nucleation density for the magnetic domains in the poled and non-poled areas. These data were processed using the formalism of Néel-Brown model, which has been previously used for analyzing the thermally-activated nucleation in similar samples with conventional (non-ferroelectric) gate. ${ }^{10}$ According to the model, the nucleation probability reads as

$$
P(t)=1-e^{-t / \tau},
$$

where the nucleation rate is

$$
\frac{1}{\tau}=\frac{1}{\tau_{0}} e^{-E_{N} / k_{B} T},
$$

and $\tau_{0}=10^{-10} \mathrm{~s}$ is the attempt rate, $\mathrm{E}_{\mathrm{N}}$ is the energy barrier, and $\mathrm{T}=300 \mathrm{~K}$ is the temperature. The nucleation density at fixed time $(\mathrm{t}=1 \mathrm{~s})$ can be obtained for varying nucleation energy $E_{N}$ through Monte-Carlo simulation. These simulation data have been matched to the experimental Kerr images in order to obtain an estimation of $\mathrm{E}_{\mathrm{N}}$.

Comparing the $\mathrm{E}_{\mathrm{N}}$ obtained as described above from poled and non-poled areas (Fig. 2(b)), we conclude that ferroelectric field effect changes $\mathrm{E}_{\mathrm{N}}$ by $3-5 \mathrm{k}_{\mathrm{B}} \mathrm{T}(\sim 0.1 \mathrm{eV})$, as shown in the inset of Fig. 2(b). This is of the same order as the $\mathrm{E}_{\mathrm{N}}$ change of $0.07 \mathrm{eV}$ in Ref. 10 obtained for the electric field of $0.3 \mathrm{~V} / \mathrm{nm}$. In addition, if we assume a nucleation volume of $(10 \mathrm{~nm})^{3}$, the MAE change is of the order of $10^{4} \mathrm{~J} / \mathrm{m}^{3}$, which is consistent with the anisotropy changes obtained in the same material for the electric field of $0.1 \mathrm{~V} / \mathrm{nm} .^{11}$

A close examination of the depletion image in Figs. 2(a) and 2(b) reveals some enhancement of switching occurring at the electrode boundaries. The exact origin of this "outlining effect" could not be identified at the present stage, however, most probably, it is linked to the electrode processing. Specifically, a possible explanation may be related to the polarization imprint occurring near the electrode boundaries.

The observed non-volatile change of the domain nucleation energy implies a change of the magnetic field required to trigger the nucleation process. To further explore this effect, we performed a series of MOKE measurements at different magnetic fields. Comparing these MOKE images, we attempted to find a pair of images, including one image from the poled and the other one from the non-poled area, where the nucleation densities are the same. This comparative analysis indicates that the nucleation density reached at $24.8 \mathrm{mT} / 50 \mathrm{~ms}$ in the accumulation state is the same as in the non-poled area at $23.0 \mathrm{mT} / 50 \mathrm{~ms}$ (Fig. 2(c)). The other pair of images in Fig. 2(c) shows that the same nucleation density is reached for the non-poled area and the area poled in the depletion state at $24.8 \mathrm{mT} / 50 \mathrm{~ms}$ and $22.4 \mathrm{mT} / 50 \mathrm{~ms}$, respectively. Thus, the effect of poling the ferroelectric gate in the accumulation/depletion state is equivalent to the nucleation energy change induced by a magnetic field variation of approximately $+/-2 \mathrm{mT}$.

The experiments described above clearly show the ferroelectric control of the magnetic domain nucleation due to the sensitivity of MAE to the electric field. The other implication of the field-effect-tuned MAE is a possibility to change the domain wall (DW) velocity. ${ }^{10-12}$ In order to directly observe the impact of the ferroelectric gate polarization on the DW propagation, we study a different area in a thinner part of the $\mathrm{Al}$ wedge resulting in a more oxidized $\mathrm{Co} / \mathrm{AlOx}$ interface and greater MAE. Due to the relatively high MAE, the switching is triggered in a very limited number of reversed domains and develops further through the expansion of these domains (as shown in Movies 3 and 4 in the supplementary material ${ }^{18}$ ). In this switching mode, the effect of the ferroelectric gate on the domain wall speed has been quantified by detecting the domain wall position vs. time under a constant magnetic field of $12 \mathrm{mT}(13 \mathrm{mT})$ for the accumulation (depletion) state. The results of this experiment are summarized in Fig. 3 (the details of DW propagation measurement are presented in the supplementary material ${ }^{18}$ ). In agreement with the results obtained in the previous experiments for similar velocities, ${ }^{10}$ DW velocity decreased by a factor 1.6 (increased by a factor 2.1) in the accumulation (depletion) states compared to the non-poled state.
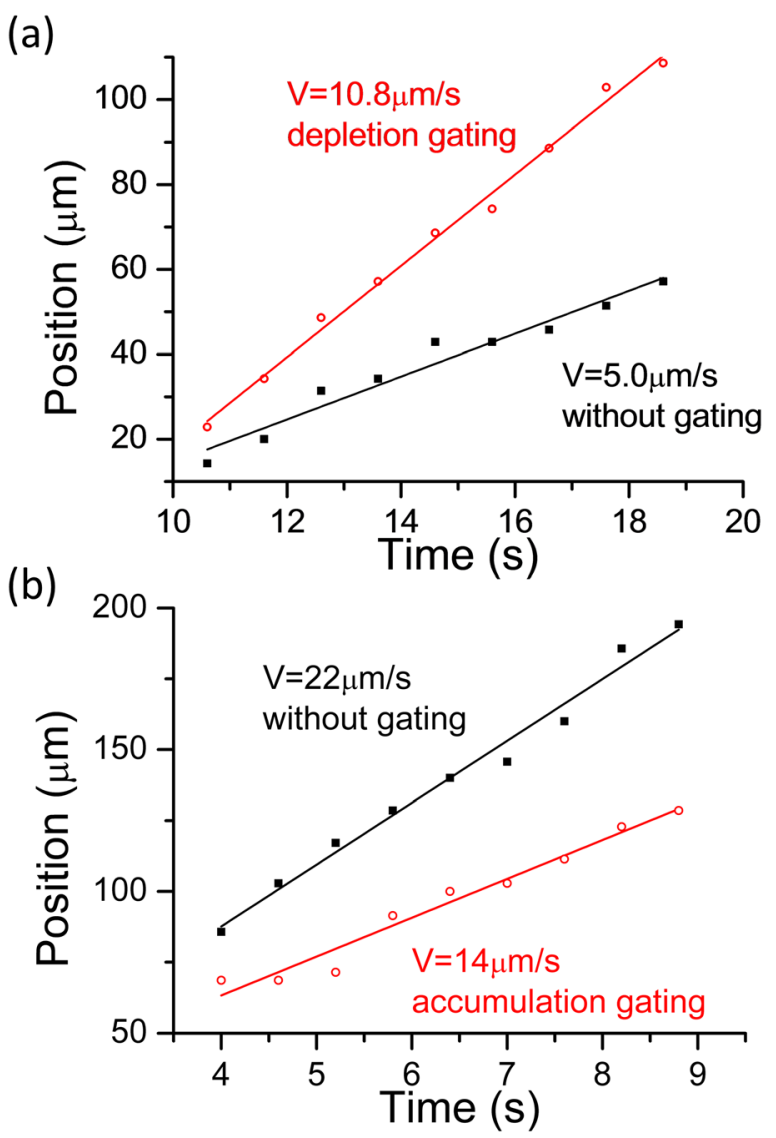

FIG. 3. Domain wall positions obtained from the series of MOKE images under a constant magnetic field of $12 \mathrm{mT}(13 \mathrm{mT})$ for the accumulation (depletion) state. (a) Accumulation state vs. non-poled area and (b) depletion state vs. non-poled area. 
Comparison of the DW velocity change induced in the identically grown magnetic layers by the ferroelectric gate and the conventional (non-ferroelectric) gate studied in Ref. 10 enables us to estimate the magnitude of the net charge associated with the spontaneous polarization. For instance, the DW velocity decrease by factor 1.6 shown in Fig. 3(a) can be compared to a similar effect achieved by applying $2 \mathrm{~V}$ to the $\mathrm{Al}_{2} \mathrm{O}_{3}(7 \mathrm{~nm}) / \mathrm{HfO}_{2}(37 \mathrm{~nm})$ gate. ${ }^{10}$ In case of this non-ferroelectric gate, the charge density induced at the interface between the $\mathrm{HfO}_{2}$ layer and magnetic channel is about $0.7 \mu \mathrm{C} / \mathrm{cm}^{2}$. Hence, for the ferroelectric P(VDF-TrFE) gate, the charge density is likely to be the same. On the other hand, polarization hysteresis measurements (Fig. 1(b)) show that the spontaneous polarization of the ferroelectric gate is close to $6 \mu \mathrm{C} / \mathrm{cm}^{2}$, i.e., almost an order of magnitude higher. This discrepancy is explained by strong screening of the spontaneous polarization charge in the layer adjacent to the ferroelectric film interface. The fundamental origin of this effect is linked to the existence of so-called "dead layer ${ }^{19}$ " at the ferroelectric film interface. The degradation of ferroelectricity in this layer results in a very high electric field occurring when the film is poled. The charge injection induced by this field causes the spontaneous polarization screening and reduces the gate effect to the values significantly weaker than the estimation based on the measured spontaneous polarization. For $\mathrm{P}(\mathrm{VDF}-\mathrm{TrFE})$ ferroelectric gates integrated with a wide range of materials, including wide-gap semiconductor heterostructures $^{20}$ and heavily doped magnetic semiconductors, ${ }^{21}$ similar effect of polarization screening has been observed. These results suggest that by improving the structural quality and dielectric strength of the interface-adjacent layers of ferroelectric films, the non-volatile gate effect can be significantly enhanced.

In conclusion, a purely electrical non-volatile control of the magnetic domain nucleation and movement has been demonstrated at room temperature for an ultra-thin layer of cobalt. The ferroelectric domains projected onto the magnetic channel change locally the magnetic anisotropy energy, resulting in significantly altered magnetic domain dynamics. This offers a potentially attractive tool for defining magnetic racetracks, nucleation spots, and pinning sites for the magnetic domains.

Co-authors from EPFL acknowledge the support from the Swiss National Science Foundation: Project
No. 200020_132724. EU is acknowledged for financial support through the projects ERC- 268058 MOBILE-W, and STSM COST-0904 program. Co-authors from Néel Institut acknowledge the support from the French National Research Agency (ANR) under the project ANR-2010 BLANC-1006-ELECMADE

${ }^{1}$ H. Ohno, D. Chiba, F. Matsukura, T. Omiya, E. Abe, T. Dietl, Y. Ohno, and K. Ohtani, Nature 408, 944 (2000).

${ }^{2}$ M. Sawicki, D. Chiba, A. Korbecka, Y. Nishitani, J. A. Majewski, F. Matsukura, T. Dietl, and H. Ohno, Nat. Phys. 6, 22 (2010).

${ }^{3}$ I. Stolichnov, S. W. E. Riester, H. J. Trodahl, N. Setter, A. W. Rushforth, K. W. Edmonds, R. P. Campion, C. T. Foxon, B. L. Gallagher, and T. Jungwirth, Nature Mater. 7, 464 (2008).

${ }^{4}$ E. Mikheev, I. Stolichnov, E. De Ranieri, J. Wunderlich, H. J. Trodahl, A. W. Rushforth, S. W. E. Riester, R. P. Campion, K. W. Edmonds, B. L. Gallagher, and N. Setter, Phys. Rev. B 86, 235130 (2012).

${ }^{5}$ M. Weisheit, S. Fähler, A. Marty, Y. Souche, C. Poinsignon, and D. Givord, Science 315, 349 (2007).

${ }^{6}$ T. Maruyama, Y. Shiota, T. Nozaki, K. Ohta, N. Toda, M. Mizuguchi, A. A. Tulapurkar, T. Shinjo, M. Shiraishi, S. Mizukami, Y. Ando, and Y. Suzuki, Nat. Nanotechnol. 4, 158 (2009).

${ }^{7}$ T. Seki, M. Kohda, J. Nitta, and K. Takanashi, Appl. Phys. Lett. 98, 212505 (2011).

${ }^{8}$ D. Chiba, S. Fukami, K. Shimamura, N. Ishiwata, K. Kobayashi, and T. Ono, Nature Mater. 10, 853 (2011).

${ }^{9}$ K. Shimamura, D. Chiba, S. Ono, S. Fukami, N. Ishiwata, M. Kawaguchi, K. Kobayashi, and T. Ono, Appl. Phys. Lett. 100, 122402 (2012).

${ }^{10}$ A. Bernand-Mantel, L. Herrera-Diez, L. Ranno, S. Pizzini, J. Vogel, D. Givord, S. Auffret, O. Boulle, I. M. Miron, and G. Gaudin, Appl. Phys. Lett. 102, 122406 (2013).

${ }^{11}$ A. J. Schellekens, A. van den Brink, J. H. Franken, H. J. M. Swagten, and B. Koopmans, Nat. Commun. 3, 847 (2012).

${ }^{12}$ Y. Shiota, S. Murakami, F. Bonell, T. Nozaki, T. Shinjo, and Y. Suzuki, Appl. Phys. Express 4, 043005 (2011).

${ }^{13}$ P. Lukashev, T. Paudel, J. López-Encarnación, S. Adenwalla, E. Tsymbal, and J. Velev, ACS Nano 6, 9745 (2012).

${ }^{14}$ A. Mardana, S. Ducharme, and S. Adenwalla, Nano Lett. 11, 3862 (2011).

${ }^{15}$ Y. Chu, L. W. Martin, M. Holcomb, M. Gajek, S. Han, Q. He, N. Balke, C. Yang, D. Lee, W. Hu, Q. Zhan, P. Yang, A. Fraile-Rodríguez, A. Scholl, S. Wang, and R. Ramesh, Nature Mater. 7, 478 (2008).

${ }^{16}$ D. Lebeugle, A. Mougin, M. Viret, D. Colson, and L. Ranno, Phys. Rev. Lett. 103, 257601 (2009).

${ }^{17}$ A. Manchon, C. Ducruet, L. Lombard, S. Auffret, B. Rodmacq, B. Dieny, S. Pizzini, J. Vogel, V. Uhlir, M. Hochstrasser, and G. Panaccione, J. Appl. Phys. 104, 043914 (2008).

${ }^{18}$ See supplementary material at http://dx.doi.org/10.1063/1.4833495 for domain growth dynamics data.

${ }^{19}$ A. K. Tagantsev, I. Stolichnov, N. Setter, and J. S. Cross, J. Appl. Phys. 96, 6616 (2004).

${ }^{20}$ L. Malin, I. Stolichnov, and N. Setter, J. Appl. Phys. 102, 114101 (2007).

${ }^{21}$ I. Stolichnov, S. Riester, E. Mikheev, N. Setter, A. Rushforth, K. Edmonds, R. Campion, T. Foxon, B. Gallagher, T. Jungwirth, and J. Trodahl, Nanotechnology 22, 254004 (2011). 